\title{
FACTORES AMBIENTALES ASOCIADOS CON LABIO O PALADAR HENDIDO NO SINDRÓMICO EN UNA POBLACIÓN DEL MAGDALENA MEDIO COLOMBIANO
}

\author{
${ }^{1}$ Andrea Melisa Prada Flórez, ${ }^{1}$ Gloria Marcela Eljach Sánchez, ${ }^{1}$ Verónica Caballero Barbosa, \\ ${ }^{2}$ Ethman Ariel Torres Murillo \\ ${ }^{1}$ Estudiantes X Semestre de Odontología, U. Santo Tomás, Colombia. \\ ${ }^{2}$ Especialista en Estomatología Pediátrica, U. Nacional de Colombia, especialista en Ortodoncia U. Santo Tomás, \\ magíster en Administración U. Santo Tomás, docente U. Santo Tomás, Colombia.
}

Autor responsable de correspondencia: Ethman Ariel Torres Murillo

Correro electrónico: ethmant@yahoo.com

\section{RESUMEN}

Objetivo: evaluar la posible asociación entre factores ambientales y la presencia de labio o paladar hendido (LPH) no sindrómico en los niños residentes en el Magdalena Medio colombiano.

Materiales y métodos: se realizó un estudio de casos y controles con 30 madres de niños con LPH (casos) y 60 madres de niños sin alguna alteración craneofacial (controles). Los casos y controles se obtuvieron de una clínica dental en la región mencionada. Las variables analizadas fueron las sociodemográficas (edad, condición socioeconómica, nivel de escolaridad, lugar de residencia antes y durante el primer trimestre); las relacionadas con la gestación (exposición a pesticidas, consumo prenatal de sustancias psicoactivas, alcohol y tabaco), la presencia de LPH en la familia. Se aplicó la prueba de $\mathrm{Chi}^{2}$ o test Exacto de Fisher según fuera apropiado, la t de Student o U. de Mann Whitnney de acuerdo con la distribución de las variables. Se realizó una regresión logística para establecer los Odds Ratio (OR) crudos y ajustados.

Resultados: se consideraron como factores protectores haber estudiado en la universidad [OR: 0,1 IC 95\%: 0,01 - 0,70] y la condición socioeconómica más alta [OR: 0,07 IC 95\%: 0,02-0,60]. Se encontró que la exposición a plaguicidas [OR: 14,3 IC 95\%: 4,3-47,8] y la presencia de LPH en la familia [OR: 5,7 IC 95\%: 1,3 - 24,9] se comportaron como factores de riesgo.

Conclusiones: la exposición a los pesticidas y la presencia de LPH en la familia del niño afectado aumentaron el riesgo de hendiduras no sindrómicas. [Prada AM, Eljach GM, Caballero V, Torres EA. Factores ambientales asociados con labio o paladar hendido no sindrómico en una población del Magdalena Medio colombiano. Ustasalud. 2014; 13: 18 - 25]

Palabras clave: fisura del paladar, labio leporino, factores de riesgo, plaguicidas.

\section{ENVIRONMENTAL FACTORS ASSOCIATED WITH ORAL CLEFTS IN CHILDREN LIVING IN COLOMBIAN MAGDALENA MEDIO}

\section{ABSTRACT}

Objective: to assess the possible association between environmental factors and the presence of non-syndromic cleft lip and/or palate $(\mathrm{CL} / \mathrm{P})$ in children living in the Colombian Magdalena Medio.

Methods: a case-control study was carried out with 30 mothers of children with CL/P (cases) and 60 mothers of children without any craniofacial alteration (controls). The cases and controls were obtained from a dental clinic in the region mentioned. The variables analyzed were sociodemographic (age, socioeconomic condition, educational level, place of residence before and during the first trimester); pregnancy background (prenatal examinations, diseases, drugs, alcohol and tobacco consumption, psychoactive substances consumption and pesticide exposure), presence of $\mathrm{CL} / \mathrm{P}$ in the family. Chi-square and Fisher Exact test, Student $\mathrm{t}$ test or $\mathrm{U}$ Mann Whitney were performed when appropriate. A logistic regression was done to establish the crude and adjusted odds ratio (OR).

Results: the educational level in college category [OR: 0.1 95\% CI: $0.01-0.70$ ] and the highest socioeconomic condition [OR: 0.07 95\% CI: $0.02-0.60$ ] were considered as protective factors for $\mathrm{CL} / \mathrm{P}$. The results in the logistic regression showed that pesticide exposure [OR: 14.3 95\% CI: 4.3 - 47.8] and the presence of CL/P in the family [OR: 5.7 95\% CI: $1.3-24.9$ ] behaved as risk factor for oral clefts. Diagnostic test for regression model showed a Hosmer-Lemershow test of $4.56(\mathrm{p}=0.1022)$ indicating a good fit of the model.

Conclusions: exposure to pesticides and the presence of $\mathrm{CL} / \mathrm{P}$ in the family of the affected child increased the risk for non-syndromic oral clefts.

Key words: cleft lip, cleft palate, congenital abnormalities, risk factors, agrochemicals. 


\section{INTRODUCCIÓN}

El labio o paladar hendido (LPH) no sindrómico es una malformación que se presenta por una falla en la fusión de los procesos maxilares y el proceso nasal interno que ocurre en las primeras doce semanas de gestación. Su etiología es controversial y multifactorial, puesto que puede ser causada por múltiples genes, generalmente de la familia Homeobox, otorgándole un carácter poligénico. Así mismo, algunos factores ambientales aún no muy bien documentados pueden intervenir en su desarrollo. Es por esto que dicha alteración se ha calificado como una anomalía multifactorial y poligénica por la mayoría de investigadores. ${ }^{1,2}$

El labio hendido se origina por una fusión incompleta de los procesos maxilares y naso medial durante el periodo embrionario, puede ser unilateral o bilateral. El paladar hendido ocurre por la falla de unión de las prominencias palatinas. ${ }^{2,3}$ En cuanto a su fisiopatología, la mayoría de autores coinciden que esta falta de fusión se produce por una alteración en la migración celular y una alteración intrínseca en los procesos de adhesión celular entre la cuarta y novena semana de vida intrauterina. ${ }^{4}$

Según la literatura, las hendiduras labiales y palatinas pueden ocurrir juntas o separadas y la prevalencia varía según la zona geográfica. El labio fisurado ocurre en 1:1000 nacidos vivos, es más frecuente en el sexo masculino mientras que el paladar hendido se presenta en 1:2500 nacidos vivos, más usual en mujeres. ${ }^{5}$ La mayoría de las veces se presenta en forma conjunta la presencia de labio y paladar. En Colombia, su prevalencia es de 1,54 por cada 1000 nacidos vivos. Esta varía según la altura sobre el nivel del mar, siendo de 0,88:1000 a nivel del mar y de 1,69:1000 en poblaciones ubicadas por encima de los 2000 metros sobre el nivel del mar. ${ }^{6}$ Un estudio realizado en dos centros hospitalarios de la ciudad de Bucaramanga (Santander) encontró una prevalencia de LPH de 1,56 por 1000 nacido vivos. ${ }^{7}$

En cuanto a los factores ambientales estudiados como etiológicos se encuentra el cigarrillo, alcohol, consumo de medicamentos y antecedentes en la fumigación, dentro de los cuales se ha reportado el glifosfato. ${ }^{8}$ Se ha sugerido que este pesticida es nocivo para el organismo humano debido a su toxicidad sobre las células placentarias; además, actúa como un disruptor endocrino en la actividad de la aromatasa y puede alterar la estructura del ADN. ${ }^{9}$

Los efectos de los fármacos sobre el feto o neonato varían, puesto que dependen principalmente del momento del embarazo en el que se administran o consumen. Durante el periodo de organogénesis, es decir, hasta las doce semanas, los fármacos conside- rados con efectos teratogénicos producen abortos 0 malformaciones congénitas. ${ }^{10}$

Dada la insuficiente investigación sobre agentes ambientales relacionados con la etiología del labio y paladar hendido en la zona del Magdalena Medio colombiano y al considerar que existen factores en esta región que pueden estar directa o indirectamente relacionados con el desarrollo de la entidad se decidió realizar la presente investigación. Los ingresos de gran parte de las personas que viven en esta región de Colombia se obtienen a partir de la agricultura, explotación minera y otros trabajos donde los padres están expuestos a sustancias como los agroquímicos.

Por tanto, el objetivo de este trabajo fue describir los factores de riesgo ambientales asociados con LPH no sindrómico en una población del Magdalena Medio colombiano.

\section{MATERIALES Y MÉTODOS}

Se realizó un estudio de casos y controles en el que la persona "caso" fue aquella que presentó hendidura labio o palatina no sindrómica y que se encontraba registrada en la Secretaría de Salud de la ciudad de Barrancabermeja. La persona "control" se definió como aquella que no presentaba la hendidura pero que compartió características de la persona "caso".

La muestra se obtuvo a partir de los casos detectados, registrados y localizados en la región del Magdalena Medio. Esta zona se ubica en la parte medio-oriental del país, se caracteriza por la explotación de hidrocarburos y algunas áreas son fumigadas con pesticidas debido a la presencia de cultivos ilegales. Los controles se obtuvieron a partir de las madres de los niños y adolescentes sin hendiduras orofaciales que asistían a recibir tratamiento odontológico en una clínica de la región en la cual también eran atendidos los "casos". Se seleccionaron dos controles por caso.

Se evaluaron las variables sociodemográficas (sexo, edad, condición socioeconómica, escolaridad, lugar y zona de residencia antes y durante el primer trimestre de embarazo). Antecedentes de la madre (controles prenatales, enfermedades durante el embarazo, consumo de medicamentos, alcohol, tabaco, sustancias psicoactivas, exposición a agroquímicos) y antecedentes hereditarios como el tipo y ubicación de la hendidura y la presencia de familiares con LPH.

Para la obtención de la información se realizó un cuestionario en el que se registraron los datos aportados por cada una de las madres. Para confirmar el correcto funcionamiento del instrumento se llevó a cabo una prueba piloto con seis casos obtenidos de la base de datos y doce controles, que eran atendidos en la clínica en mención. 


\section{Protocolo para el procedimiento de la prueba de campo}

Se concertaron las reuniones con las madres de los menores luego de obtener su consentimiento para participar en el estudio. El cuestionario fue diligenciado mediante entrevista de manera que se pudieran aclarar las dudas en relación con las preguntas.

Al obtener la información, se crearon bases de datos en Excel que posteriormente fueron validadas y exportadas al paquete estadístico Stata.

\section{Análisis estadístico}

Se realizó la descripción de las variables de acuerdo con su naturaleza. Para las variables cualitativas, se calcularon proporciones y para las cuantitativas, medidas de tendencia central. Posteriormente, las variables cualitativas fueron analizadas con una prueba de Chi cuadrado o Test Exacto de Fisher, según fuera apropiado. Las cuantitativas se analizaron con t de Student o U. Mann Whitney según su distribución. Para establecer la asociación entre la presencia de LPH y los factores ambientales se realizó una regresión logística para obtener los Odds Ratio (OR) crudos y ajustados.

El modelamiento fue paso a paso hacia adelante. Para este se eligieron las variables que presentaron un valor de $\mathrm{p}<0,20$ en el análisis bivariado (Tabla 4). El modelo inició con las variables de menor valor $\mathrm{p}$ y, posteriormente, se incluyeron a la regresión una a una las demás variables. La decisión de mantener la variable en el modelo partió del cambio en el $10 \%$ en el coeficiente de la variable explicitoria principal (exposición a pesticidas) y el valor $\mathrm{p}$ de la variable estudiada. ${ }^{11}$ No se incluyeron variables de manera forzada en el modelo debido a las limitaciones en la selección de los sujetos tal y como se mencionó anteriormente.

\section{Consideraciones éticas}

Este trabajo se acogió a las normas establecidas en la Resolución No. 008430 de 4 de octubre de 1993, mediante la cual se clasificó como una "Investigación sin Riesgo". Se garantizaron los principios de beneficiencia, equidad, confidencialidad y veracidad en el manejo de los datos y se obtuvo el consentimiento informado de cada una de las madres participantes.

\section{RESULTADOS}

\section{Análisis univariado}

Al evaluar la totalidad de la población de estudio se encontró que el $52,2 \%$ de los menores con y sin LPH eran de sexo masculino, el $82,2 \%$ tenían menos de 12 años y el 50\% entre los 6 y 12 años. La edad materna en el momento de quedar en gestación de estos niños(as) fue de 15 a 25 años en el $64,4 \%$ y el $46,7 \%$ se encontraba realizando estudios de secundaria. Así mismo, el 68,9\% de las personas pertenecían a los estratos socioeconómicos uno y dos (Tabla 1).

Con respecto al nivel de escolaridad, haber realizado estudios en la universidad presentó una diferencia estadísticamente significativa y el valor de OR se consideró como protector [OR: 0,1 IC 95\%: 0,01 $-0,70 p=0,0064$ y en el estrato socioeconómico cuatro también evidenció una diferencia estadísticamente significativa y el $\mathrm{OR}$ resultó menor que 1,0 [OR: 0,07 IC 95\%: 0,02-0,60 p=0,030] (Tabla 1).

En relación con el lugar y la zona de residencia se encontró que la zona rural obtuvo una diferencia estadísticamente significativa, tanto antes de la gestación como durante el primer trimestre [OR 13,0 IC $95 \%$ : $4,0-43,4 \mathrm{p}<0,0000$ y OR 32,7 IC $95 \%: 8,0$ $-152,7 \mathrm{p}<0,0000$, respectivamente] (Tabla 2).

En cuanto a los antecedentes maternos evaluados, solamente hubo una diferencia estadísticamente significativa, la exposición a pesticidas durante el primer trimestre con un OR de 12,6 [IC 95\%: 3,5 $49,9]$ y la presencia de familiares con LPH con un OR de 4,3 [IC 95\%: 1,0 - 21,4] (Tabla 3).

\section{Análisis estratificado}

Se realizó un análisis estratificado por el método de Mantel y Haenszel al identificar la zona de residencia y el municipio como variables potencialmente confusoras (Tabla 4). Es importante aclarar que estas son explicables con base en la metodología empleada para seleccionar los casos y los controles, pues se presentó un sesgo de selección, dado que los participantes se escogieron de manera intencionada y direccionada en la región, al influir la presencia de mayor población en las zonas urbanas, lo que hizo que los casos se concentraran preferiblemente en los municipios aledaños y los controles en Barrancabermeja.

\section{Análisis multivariado}

Aunque algunas variables (nivel de escolaridad, condición socioeconómica, zonas de residencia antes y durante la gestación, municipios de residencia antes y durante la gestación, exposición a pesticidas y antecedentes familiares) obtuvieron un valor de $\mathrm{p}<0,05$, solamente tres mostraron una diferencia estadísticamente significativa en el modelo final. Además, no se encontraron interacciones en las variables evaluadas (Tablas 5 y 6 ).

Se evaluó el modelo final con la presencia de las tres variables resultantes (Tabla 5) y el resultado de la 


\section{ARTÍCULO DE INVESTIGACIÓN CIENTÎFICA Y TECNOLÓGICA}

evaluación del ajuste no fue adecuada (test de Hosmer-Lemeshow $\mathrm{p}=0,0346$ ) optándose por el modelo más parsimonioso que explicaba el desenlace, es decir, con dos variables cuya valoración de la bondad del ajuste fue satisfactoria (test de HosmerLemeshow $p=0,1022$ ).

Tabla 1. Características sociodemográficas asociadas con LPH en la población de estudio

\begin{tabular}{|c|c|c|c|c|c|}
\hline Variables & $\begin{array}{c}\text { Con LPH } \\
\text { n (\%) }\end{array}$ & $\begin{array}{c}\text { Sin LPH } \\
\mathbf{n}(\%)\end{array}$ & OR & IC $95 \%$ & $\mathbf{P}$ \\
\hline \multicolumn{6}{|l|}{ Sexo } \\
\hline Femenino & $14(46,7)$ & $29(48,3)$ & & & \\
\hline Masculino & $16(53,3)$ & $31(51,7)$ & 1,06 & $0,4-2,8$ & 0,8814 \\
\hline \multicolumn{6}{|l|}{ Edad materna } \\
\hline 15 a 25 años & $21(70,0)$ & $37(61,7)$ & & & \\
\hline 26 a 36 años & $6(20,0)$ & $21(35,0)$ & 0,5 & $0,1-1,6$ & 0,1973 \\
\hline 37 a 47 años & $3(10,0)$ & $2(3,3)$ & 2,6 & $0,3-33,5$ & 0,2932 \\
\hline \multicolumn{6}{|l|}{ Nivel de escolaridad } \\
\hline Primaria & $11(36,7)$ & $13(21,7)$ & & & \\
\hline Bachillerato & $16(53,3)$ & $26(43,3)$ & 0,7 & $0,2-2,3$ & 0,5385 \\
\hline Universidad & $2(6,7)$ & $21(35,0)$ & 0,1 & $0,0-0,7$ & 0,0064 \\
\hline Ninguna & $1(3,3)$ & $0(0,0)$ & & & \\
\hline \multicolumn{6}{|l|}{ Estrato socio-económico } \\
\hline Uno & $17(56,7)$ & $19(31,7)$ & & & \\
\hline Dos & $10(33,3)$ & $16(26,7)$ & 0,7 & $0,2-2,2$ & 0,4924 \\
\hline Tres & $2(6,7)$ & $4(6,6)$ & 0,6 & $0,1-4,5$ & 0,5269 \\
\hline Cuatro & $1(3,3)$ & $16(26,7)$ & 0,1 & $0,0-0,6$ & 0,0030 \\
\hline Cinco & $0(0,0)$ & $5(8,3)$ & & & \\
\hline
\end{tabular}

OR: Odds Ratio. IC 95\%: Intervalo de confianza del 95\%.

Tabla 2. Zona y lugar de residencia asociados con LPH en la población de estudio

\begin{tabular}{|c|c|c|c|c|c|}
\hline Zona y lugar de residencia & $\begin{array}{c}\text { Con LPH } \\
\text { n (\%) }\end{array}$ & $\begin{array}{c}\text { Sin LPH } \\
\text { n (\%) }\end{array}$ & OR & IC $95 \%$ & $\mathbf{P}$ \\
\hline \multicolumn{6}{|l|}{ Zona de residencia antes del embarazo } \\
\hline Urbana & $10(33,3)$ & $52(86,7)$ & & & \\
\hline Rural & $20(66,7)$ & $8(13,3)$ & 13,0 & $4,0-43,4$ & 0,0000 \\
\hline \multicolumn{6}{|c|}{ Municipio de residencia antes del embarazo } \\
\hline Barrancabermeja & $10(33,3)$ & $45(75,0)$ & & & \\
\hline Otros municipios & $20(66,7)$ & $15(25,0)$ & 6,0 & $2,1-17,6$ & 0,0001 \\
\hline \multicolumn{6}{|l|}{ Zona de residencia durante el embarazo } \\
\hline Urbana & $9(30,0)$ & $56(93,3)$ & & & \\
\hline Rural & $21(70,0)$ & $4(6,7)$ & 32,7 & $8,0-152,7$ & 0,0000 \\
\hline \multicolumn{6}{|c|}{ Municipio de residencia durante el embarazo } \\
\hline Barrancabermeja & $10(33,3)$ & $53(88,3)$ & & & \\
\hline Otros municipios & $20(66,7)$ & $7(11,7)$ & 15,1 & $4,5-52,8$ & 0,0000 \\
\hline
\end{tabular}


Tabla 3. Antecedentes maternos asociados con LPH en la población de estudio

\begin{tabular}{|c|c|c|c|c|c|}
\hline Antecedentes & $\begin{array}{c}\text { Con LPH } \\
\text { n (\%) }\end{array}$ & $\begin{array}{c}\text { Sin LPH } \\
\mathrm{n}(\%)\end{array}$ & OR & IC $95 \%$ & $\mathbf{P}$ \\
\hline \multicolumn{6}{|l|}{ Control prenatal } \\
\hline Sí & $27(90,0)$ & $53(88,3)$ & & & \\
\hline No & $3(10,0)$ & $7(11,7)$ & 0,8 & $0,1-4,1$ & 0,8125 \\
\hline \multicolumn{6}{|c|}{ Enfermedades en el primer trimestre } \\
\hline No & $28(93,3)$ & $56(93,3)$ & & & \\
\hline Sí & $2(6,7)$ & $4(6,7)$ & 1,0 & $0,1-7,5$ & 1,0000 \\
\hline \multicolumn{6}{|c|}{ Ingesta de medicamentos primer trimestre } \\
\hline Sí & $22(73,3)$ & $43(71,7)$ & & & \\
\hline No & $8(26,7)$ & $17(28,3)$ & 1,1 & $0,4-3,4$ & 0,8678 \\
\hline \multicolumn{6}{|l|}{ Tabaquismo } \\
\hline No & $28(93,3)$ & $57(95,0)$ & & & \\
\hline Sí & $2(6,7)$ & $3(5,0)$ & 1,4 & $0,1-12,5$ & 0,7449 \\
\hline \multicolumn{6}{|l|}{ Alcohol } \\
\hline No & $29(96,7)$ & $55(91,7)$ & & & \\
\hline Sí & $1(3,3)$ & $5(8,3)$ & 0,4 & $0,0-3,6$ & 0,3700 \\
\hline \multicolumn{6}{|l|}{ Pesticidas } \\
\hline No & $14(46,7)$ & $55(91,7)$ & & & \\
\hline Sí & $16(53,3)$ & $5(8,3)$ & 12,6 & $3,5-49,9$ & 0,0000 \\
\hline \multicolumn{6}{|l|}{ Antecedentes familiares } \\
\hline No & $23(76,7)$ & $56(93,3)$ & & & \\
\hline Sí & $7(23,3)$ & $4(6,7)$ & 4,3 & $1,0-21,4$ & 0,0229 \\
\hline
\end{tabular}

OR: Odds Ratio. IC 95\%: Intervalo de confianza del 95\%.

Tabla 4. Análisis estratificado (Mantel y Haenszel) de las variables para incluir en el modelo con $\mathrm{p}<0,20$ en el análisis bivariado (Odds Ratio*)

\begin{tabular}{lccccc}
\hline \multicolumn{1}{c}{ Variable } & OR Crudo & IC 95\% & $\begin{array}{c}\text { OR } \\
\text { Ajustado }\end{array}$ & IC 95\% & P \\
\hline Edad materna & 12,6 & $3,5-49,9$ & 11,6 & $3,6-37,6$ & 0,7333 \\
Nivel de escolaridad & 12,6 & $3,5-49,9$ & 10,0 & $2,9-34,3$ & 0,4308 \\
Estrato socioeconómico & 12,6 & $3,5-49,9$ & 8,1 & $2,4-27,1$ & 0,3476 \\
Zona residencia antes embarazo & 12,6 & $3,5-49,9$ & 6,7 & $1,8-24,2$ & 0,4840 \\
Municipio antes embarazo & 12,6 & $3,5-49,9$ & 10,5 & $3,0-36,6$ & 0,1993 \\
Zona residencia durante embarazo & 12,6 & $3,5-49,9$ & 3,8 & $0,9-15,3$ & 0,2309 \\
Municipio durante embarazo & 12,6 & $3,5-49,9$ & 6,4 & $1,7-24,6$ & 0,3073 \\
Antecedentes familiares & 12,6 & $3,5-49,9$ & 10,7 & $3,2-35,4$ & 0,0714 \\
\hline
\end{tabular}

*No se incluyen intervalos de confianza de los OR. OR: Odds Ratio.

Tabla 5. Modelo final con tres variables

\begin{tabular}{lccc}
\hline \multicolumn{1}{c}{ Variable } & OR & IC 95\% & P \\
\hline Pesticidas & 7,4 & $2,0-28,1$ & 0,003 \\
Antecedentes familiares & 6,1 & $1,2-30,0$ & 0,026 \\
Zona de residencia antes del embarazo & 9,2 & $2,8-30,8$ & 0,000 \\
\hline
\end{tabular}

OR: Odds Ratio. IC 95\%: Intervalo de confianza del 95\%. 
ARTÍCULO DE INVESTIGACIÓN CIENTÎFICA Y TECNOLÓGICA

Tabla 6. Modelo final con dos variables

\begin{tabular}{lccc}
\hline \multicolumn{1}{c}{ Variable } & OR & IC 95\% & P \\
\hline Pesticidas & 14,3 & $4,3-47,8$ & 0,000 \\
Antecedentes familiares & 5,7 & $1,3-24,9$ & 0,021 \\
\hline
\end{tabular}

OR: Odds Ratio. IC 95\%: Intervalo de confianza del 95\%.

\section{DISCUSIÓN}

En el presente estudio se encontró que la exposición a pesticidas y los antecedentes de LPH en la familia tuvieron una asociación estadísticamente significativa con la variable de salida (presencia de LPH). No se observó una diferencia estadísticamente significativa entre la edad materna categorizada de las madres con hijos/as con LPH y las madres con hijos/as sin LPH.

No obstante, Torres y Evaristo encontraron una razón de riesgos (RR) de 8,4 entre las mamás mayores de 30 años, frente las de 20 a 30 años lo que implica que sería un factor predisponente, es decir, una madre mayor a 30 años tiene 8,4 veces el riesgo de tener un niño con hendidura labial o palatina que una madre entre los 20 a 30 años. ${ }^{12}$ Sin embargo, estos resultados no coinciden con los observados por Sotomayor y Gutarra (2004) que sugieren que la mayor cantidad de casos se presenta no a una edad muy avanzada, sino a una edad intermedia (21-30 años). ${ }^{13} \mathrm{~A}$ su vez, se encontró un segundo estudio entre las menores de 20 años frente a las de 20 a 30 años, en el que mostraban un OR de 3,9, lo que significa que sería un factor predisponente, o sea, una madre menor a 20 años tendría 3,9 veces la probabilidad de tener un niño/a con LPH que una madre mayor de esta edad. Este hallazgo no sorprende porque se ha reportado que madres muy jóvenes pueden tener complicaciones en el embarazo y una de estas es un neonato con alguna patología..$^{13,14}$

La variable "ingesta de medicamentos" no resultó ser estadísticamente significativa dado posiblemente, a que los tipos de medicamentos que fueron reportados por las madres no representaban un riesgo durante el embarazo. Sin embargo, Nazer y colaboradores (1995) registraron que los anticoagulantes y anticonvulsivantes actúan como teratógenos en la formación de fisuras bucales. ${ }^{15}$ Igualmente, Báez y colaboradores (2000) sugirieron que la ingesta de medicamentos por parte de la madre durante la gestación aumenta el riesgo cinco veces más para que se presente LPH en relación con las madres que no ingieren medicamentos durante su embarazo. ${ }^{16}$

Con respecto a las variables "consumo de alcohol" y "tabaquismo" no se encontró una asociación estadísticamente significativa similar a lo observado en el trabajo de Torres y colaboradores en una población de Bucaramanga. ${ }^{17}$ No obstante, Molina-Solana y colaboradores realizaron un meta-análisis y mostraron que el consumo de tabaco $(\mathrm{OR} 1,48)$ y alcohol (OR 1,28$)$ es considerado como factores de riesgo para el desarrollo de LPH. ${ }^{8}$ Esta asociación se incrementa al aumentar el número de cigarrillos por día, como lo mostró otra investigación en la que se incrementaba el riesgo cuando la madre fumaba de 1 a 9 cigarrillos o más de 20 cigarrillos. ${ }^{18}$ Así mismo, varios estudios han reportado que al incrementar el consumo de alcohol y cigarrillo durante los tres primeros meses de gestación aumenta el riesgo de presentar hendiduras orales. ${ }^{19-21}$

Algunas variables explicatorias (nivel de escolaridad, condición socioeconómica, zona y municipio de residencia antes y durante la gestación) se asociaron al desenlace en el análisis bivariado, sin embargo, en el modelamiento no se obtuvo significancia estadística. En el estudio realizado por Acuña y Carlo en una población mexicana se encontró asociación entre la posición socioeconómica con el LPH. ${ }^{22}$ Cuando se estudian los defectos orofaciales, se ha observado de manera consistente que las condiciones desfavorables de la posición socioeconómica se asocian con LPH como lo refieren Clark y colaboradores, ${ }^{23}$ Puho y colaboradores, ${ }^{24}$ Carmichael y colaboradores. ${ }^{25}$ En todos estos estudios se observó lo que se ha denominado el gradiente social en salud que señala que las desigualdades en la distribución del estado de salud de la población están relacionadas con las desigualdades en el estatus social. ${ }^{22-25}$ Las personas de menor posición socioeconómica presentan mayor riesgo de tener $\mathrm{LPH}^{23}$

Se pudo establecer un modelo explicativo de tres variables (incluye la zona de residencia antes del embarazo). No obstante, la evaluación de la bondad de ajuste permitió optar por un modelo que explicara el desenlace en forma más adecuada y presentara una mejor prueba de bondad de ajuste, además de ser más parsimonioso por tener solamente dos variables.

Es importante señalar que las variables zona y municipio de residencia antes del embarazo y durante la gestación, inicialmente, resultaron significativas debido a limitaciones en la forma cómo se seleccionaron los casos y los controles, punto siempre álgi- 
do en este tipo de diseños de investigación. Estas variables se presentan como colineales y además confusoras por la misma razón, es decir, expresan el resultado de una búsqueda direccionada de casos con sus respectivos controles en una región del departamento y muestran un resultado que no refleja la realidad y que va de la mano con la exposición a pesticidas y otras variables no evaluadas, propias de las personas residentes en las zonas rurales que desempeñan labores agrícolas. Según Nivia (2000) el aire, el agua y el polvo casero están altamente contaminados en las zonas rurales. Las partículas de suelo con plaguicida adherido procedente de suelos inadecuadamente protegidos pueden ser transportadas por el viento a grandes distancias, pero su destino son las viviendas ubicadas en las zonas rurales donde pueden persistir mayor tiempo al quedar protegidos del sol, la lluvia y la actividad microbiana. Estos residuos constituyen una fuente importante de contaminación para los niños y niñas desde su periodo de gestación. ${ }^{26}$

Los hallazgos de este estudio permitieron establecer un modelo que explica en forma adecuada la presencia de labio y paladar hendido que incluye la exposición a pesticidas durante el primer trimestre de embarazo con un OR de 14,3. Diversos autores han vinculado la exposición a plaguicidas durante el embarazo con el bajo peso de los neonatos, los defectos de nacimiento y el aumento en la tasa de abortos espontáneos, incluyen dentro de los defectos de nacimiento el labio o paladar hendido. ${ }^{27-30}$ Otro factor de riesgo asociado con la exposición de pesticidas puede verse relacionado con la exposición laboral de padres a agentes químicos utilizados en esta región como el glifosato, muy usado en actividades de fumigación. Carrasco y colaboradores mostraron cómo el glifosfato afectaba la morfogénesis al alterar el metabolismo del ácido retinoico. ${ }^{31}$ Estas fumigaciones se presentaron en la zona de estudio antes del 2012 según cifras determinadas por la Policía Nacional en la región del Magdalena Medio.

Igualmente, el modelo incluye la presencia de familiares con patologías relacionadas con un OR de 5,7 lo que indica que sería un factor predisponente, es decir, que un neonato con antecedentes familiares de LPH tiene 5,7 la probabilidad de presentar hendidura labial o palatina en comparación con un neonato sin estos antecedentes. Este hallazgo coincide con lo encontrado por Sacsaquispe y colaboradores, ${ }^{29}$ y Sotomayor y Gutarra. ${ }^{13}$

El glifosato es un agrotóxico usado como herbicida. Se han mostrado datos que apuntan contundentemente a una asociación clara entre la exposición al glifosato y la creciente aparición de malformaciones al nacer (teratogenias) que afectan la formación de la cresta neural, los patrones romboidales y la diferenciación neural primaria, además de efectos en la fertilidad y abortos anómalos en las poblaciones humanas que están cerca de los campos donde se aplica el glifosato. La frecuencia de las teratogenias aumentó conforme las concentraciones de glifosato en el medio de cultivo y en experimentos de microinyección también se incrementaron. ${ }^{30,31}$

Así mismo, Paganelli y colaboradores mencionaron que las concentraciones que utilizaron en sus experimentos son mucho menores a las usadas en los cultivos transgénicos o las que se han encontrado acumuladas en agua o suelos, y probablemente las halladas en el aire que son aspiradas por las personas que viven cerca de los cultivos transgénicos 0 incluso son menores que las que se registran en fluidos y tejidos humanos y particularmente fetales. ${ }^{30}$

Es importante relacionar este tipo de estudios con investigaciones genéticas, así también es necesario implementar estrategias de vigilancia epidemiológica que permitan conocer la magnitud del problema en el ámbito regional y nacional para establecer políticas públicas de educación, prevención y control.

\section{BIBLIOGRAFÍA}

1. Bianchi F, Calzdari E, Cordier S. Environment and genetics in the etiology of cleft lip and cleft palate. Epidemiol Prev. 2000; 24: 21-27.

2. Farronato G, Cannalire P, Martinelli G. Cleft lip and palate: review. Minerva Stomatol. 2014; 63: 111-126.

3. Kapadia H, Mues G, Souza R. Genetics and human malformations. Craniofacial Res. 2007; 10: 105-113.

4. Nassif A, Senussi I, Meary F, Loiodice S. Msx1 role in craniofacial bone morphogenesis. Bone. 2014; 66: 96-104.

5. Aizenbud D, Coval M, Harari D. Isolated soft tissue cleft lip: epidemiology and associated dental anomalies. Oral Dis. 2011; 17: 221-231.

6. García J, Caro M. Epidemiologia y factores de riesgo en pacientes con hendiduras orales en poblaciones colombianas ubicadas a una altitud superior a los 200 metros sobre el nivel del mar. Acta Otorrinolaringol Cir Cabeza Cuello. 2009; 37: 139-147.

7. Serrano CA, Ruíz JM, Quiceno LF, Rodríguez MJ. Prevalencia de labio y/o paladar hendido en dos instituciones hospitalarias de la ciudad de Bucaramanga entre enero de 2005 y septiembre de 2009 [Trabajo de Grado]. Bucaramanga: Universidad Santo Tomás; 2010.

8. Molina-Solana R, Yáñez-Vico RM, Iglesias-Linares A, Mendoza-Mendoza A, Solano-Reina E. Current concept on the effect of environmental factors on cleft lip and palate. Int J Oral Maxillofac Surg. 2013; 42: 177-184.

9. Burger M, Fernández S. Exposición al herbicida glifosato: aspectos clínicos toxicológicos. Rev Med Uruguay. 2004; 20: 202-207.

10. Larrubia O, Pérez F. Fármacos y embarazo [en línea] 2010; 


\section{ARTÍCULO DE INVESTIGACIÓN CIENTÍFICA Y TECNOLÓGICA}

URL disponible en: http://www.jano.es/ficheros/sumarios/1/0/1762/66/00660071_LR.pdf

11. Greenland S. Modeling and variable selection in epidemiologic analysis. Am J Public Health. 1989; 79: 340-349.

12. Torres M, Evaristo T. Factores de riesgo de labio y paladar fisurado en neonatos Hospital Nacional San Bartolome periodo 2000-2009. Odontol Sanmarquiana. 2001; 14: 1214.

13. Sotomayor J, Gutarra L. Factores de riesgo en el embarazo para la presencia de fisuras labio máxilo palatinas. Odontol Sanmarquina. 2004; 8: 17-24.

14. Sepúlveda G, Palomino H, Cortés J. Prevalencia de fisura labio palatino e indicador de riesgo: Estudio de la población atendida en el Hospital Clínico Félix Bulnes de Santiago de Chile. Rev Esp de Cirug Oral y Máxilofac. 2008; 30: 17-25.

15. Nazer HJ, Villa VJJ, Van Deer Baars MR, Cifuentes OL. Incidencia de labio leporino y paladar hendido en Latinoamérica: periodo 1982-1990. Pediatría. 1995; 37: 13-19.

16. Báez R, Bastidas R, Monserat E. Labio y paladar hendido. Act Odontol Venez. 2000; 38: 15-20.

17. Torres EA, Gómez G, Pinzón Z. Asociación entre el consumo de cigarrillo y alcohol en la gestante como factor de riesgo para labio y/o paladar hendido no sindrómico. Ustasalud. 2012; 11: 88-94.

18. Lie RT, Wilcox AJ, Taylor J. Maternal smoking and oral clefts. Epidemiology. 2008; 19: 606-615.

19. Romitti PA, Sun L, Correa A. Maternal periconceptional alcohol consumption and risk for orofacial clefts. Am J Epidemiology. 2007: 134: 298-303.

20. Leite IC, Koifman S. Oral clefts, consanguinity, parenteral tobacco an alcohol use: a case-control estudy in Rio de Janeiro. Braz Oral Res. 2009; 23: 31-37.

21. Grewal J, Ma C, Lammer EJ. Maternal periconceptional smoking and alcohol consumption and risk for select congenital anomalies. Birth Defects Res. 2008; 82: 519526.

22. Acuña G, Carlo E, Medina S. Factores de riesgo hereditarios y socioeconómicos para labio y paladar hendido en México: casos y controles. Biomédica. 2011; 31: 343-350.

23. Clark JD, Mossey PA, Sharp L, Little J. Socioeconomic status and orofacial clefts in Scotland, 1989 to 1998. Cleft Palate Craniofacial J. 2003; 40: 481-485.

24. Puho E, Metneki J, Czeizel AE. Maternal employment status and isolated orofacial clefts in Hungary. Cent Eur J Public Health. 2005; 13: 144-148.

25. Carmichael SL, Nelson V, Shaw GM, Wasserman CR, Croen LA. Socio-economic status and risk of conotruncal heart defects and orofacial clefts. Paediatr Perinat Epidemiol. 2003; 17: 264-271.

26. Nivia, E. Mujeres y plaguicidas. Una mirada a la situación actual, tendencias y riesgos de los plaguicidas. Rapalmira, Colombia; 2000. URL disponible en http://www.rap-al. org/articulos_files/Mujeres\%20y\%20Plaguicidas.pdf

27. Vanderas AP. Incidence of cleft lip, cleft palate, and cleft lip and palate among races: A review. Cleft Palate J. 1987; 24:216-25

28. Palomino HM, Palomino H, Cauvi D, Barton SA, Chakraborty R. Facial clefting and amerindian admixture in populations of Santiago, Chile. Am J Hum Biol. 1997; 9: 225-232.
29. Sacsaquispe S, Ortiz L. Prevalencia de labio y/o paladar fisurado y factores de riesgo. Rev Estomatol Herediana. 2004; 14: 54-58.

30. Paganelli A, Gnazzo V, Carrazco A. Herbicidas a base de glifosfato producen efectos teratogénicos en vertebrados interfiriendo en el metabolismo ácido retinoico. Chem Res Toxicol. 2010; 23.

31. Carrasco A. Evaluación de la información científica vinculada al glifosato en su incidencia sobre la salud humana y el ambiente [en línea] Ciudad Autónoma de Buenos Aires; 2009. URL disponible en: http://www.msal.gov.ar/agroquimicos/pdf/INFORME-GLIFOSATO-2009-CONICET.pdf

\section{Correo electrónico de los autores:}

Andrea Melisa Prada Flórez: andreitaprada08@hotmail.com Gloria Marcela Eljach Sánchez: marce125@hotmail.com Verónica Caballero Barbosa: verito.č @ hotmail.com Ethman Ariel Torres Murillo: ethmant@yahoo.com 